\title{
A CANONICAL CONNECTION ASSOCIATED WITH CERTAIN G-STRUCTURES
}

\author{
José M. Sierra and Antonio Valdés, Madrid
}

(Received June 9, 1994)

\section{INTRODUCTION}

The problem of finding differential invariants of $G$-structures leads to the problem of finding natural connections associated to $G$-structures. Let us explain how it goes in the case of Riemannian manifolds. Let $M$ be a differentiable manifold. Suppose that there exists a scalar function $F=F\left(j_{x}^{r} g\right)$ defined over the manifold of $r$-jets of Riemannian metrics on $M$ with the following property: If $f: M \longrightarrow M$ is a diffeomorphism then $F\left(j_{f(x)}^{r} f^{*} g\right)=F\left(j_{x}^{r} g\right)$. Such a function is said to be a differential invariant. Examples of such functions are the Weyl invariants, which are the functions obtained by taking the successive covariant derivatives of the curvature $R$ of the metric, taking tensor products and contracting in all possible ways:

$$
\operatorname{trace}\left(\nabla^{l_{3}} R \otimes \cdots \otimes \nabla^{l_{m}} R\right)
$$

It is a well known result that the Weyl invariants generate all the polynomial invariants (see [1] and [4]).Taking into account this result, it is natural to approach the problem of finding the differential invariants of an arbitrary $G$-structure in that of finding a natural connection attached to the $G$-structure like the Levi-Civita connection is attached to the Riemannian structures, i.e., functorial with respect the action of the group of diffeomorphisms of the base manifold and adapted to the $G$-structure. The connection must also depend only on the first contact of the $G$-structure, i.e., the connection must not loose information of the $G$-structure. This is the aim of this paper. ${ }^{1}$

Briefly our main theorem says that if the Lie algebra $\mathfrak{G}$ of $G$ satisfies certain algebraic condition then there exists a unique connection $\nabla^{P}$ adapted to each $G$ structure $P \rightarrow M$ and attached in a functorial way which is determined by the

\footnotetext{
${ }^{1}$ For a recent exposition on natural geometric objects see [7]
} 
following condition: For every section $S$ of the adjoint bundle of endomorphisms associated to the principal bundle of the $G$-structure and for every vector field $X$ of $M$

$$
\operatorname{trace}\left(S \circ i_{X} \operatorname{Tor} \nabla^{P}\right)=0 \text {. }
$$

The algebraic condition on $\mathfrak{G}$ holds immediately if the first prolongation $\mathfrak{G}^{(1)}$ of $\mathfrak{G}$ vanishes and $\mathfrak{G}$ is invariant under matrix transposition. Hence it holds if $G$ is a subgroup of the orthogonal group as a particular case. Of course we obtain the LeviCivita connection if $G$ is the orthogonal group. However in general the connection obtained is not symmetric. This is not a surprise, since in general a $G$-structure is not 1-integrable. We also find that the condition $\mathfrak{G}^{(1)}=0$ is a necessary condition for the existence a functorial connection. This explains why there exists no canonical linear connection attached to the conformal or symplectic structures. In the last section this canonical connection is explicitly computed in the almost Hermitian and almost metric contact cases. In the paper [3] all the functorial connections attached to an almost Hermitian structure are computed using techniques completely different from those of this paper. So our paper points out one of these connections as a very particular one.

The authors wish to express their sincere thanks to Prof. D. Blair. Also the first author wishes to express his sincere thanks to his thesis advisor, Prof. J. Muñoz.

\section{THE MAIN RESULT}

Let $G$ be a subgroup of the full general linear group $G L(n, \mathbb{R})$. Let $\pi: P \longrightarrow$ $M$ be a $G$-structure, and let $\nabla$ be a connection on $P$. We denote by $B(\nu)$ the standard horizontal vector field corresponding to $\nu \in \mathbb{R}^{n} ;\left(\widetilde{X}_{1}, \ldots, \widetilde{X}_{n}\right)$ is said to be the canonical basis of standard horizontal vector fields where $\widetilde{X}_{i}=B\left(e_{i}\right)$ and $\left\{e_{1}, \ldots, e_{n}\right\}$ is the canonical basis of $\mathbb{R}^{n}$ (see [6]).

We denote by $\theta$ the structure form of the $G$-structure, defined by

$$
\theta\left(X_{u}\right)=\left(\lambda^{1}, \ldots, \lambda^{n}\right)
$$

where $\pi_{\star}\left(X_{u}\right)=\sum_{k} \lambda^{k}\left(X_{k}\right)_{x}$, and $\left(X_{x}^{1}, \ldots, X_{x}^{n}\right)=u$. Let $\Theta=\left(\Theta^{1}, \ldots, \Theta^{n}\right)$ be the torsion form of $\nabla$. Let us denote by $T$ the torsion tensor field of $\nabla$, and by $\left(\omega^{1}, \ldots, \omega^{n}\right)$ the dual basis of $u \in P$. Then

$$
T^{i}\left(X_{x}^{j}, X_{x}^{k}\right)=\omega^{i}\left(T\left(X_{x}^{j}, X_{x}^{k}\right)\right)=\Theta_{u}^{i}\left(\tilde{X}_{j}, \tilde{X}_{k}\right)
$$

We denote by $\mathfrak{G}$ the Lie algebra of $G$. We will denote by Ad.P the adjoint bundle of the total space of a $G$-structure, $P$. A section of Ad.P is an endomorphism of $T M$. The notation $\operatorname{Tor}(\nabla)$ stands for the torsion tensor field of the connection $\nabla$. 
The Lie algebra $\mathfrak{G}$ of $G$ may be seen as a subalgebra of $\operatorname{gl}(n, \mathbb{R})=\operatorname{Hom}\left(\mathbb{R}^{n}, \mathbb{R}^{n}\right)$. If $T \in \operatorname{Hom}\left(\mathbb{R}^{n}, \mathfrak{G}\right), \operatorname{alt}(T) \in \Lambda^{2} \mathbb{R}^{n^{*}} \otimes \mathbb{R}^{n}$ is the torsion-type tensor given by

$$
\operatorname{alt}(T)(u, v)=T(u)(v)-T(v)(u),
$$

where $u, v \in \mathbb{R}^{n}$. The vector subspace given by all the $T \in \operatorname{Hom}\left(\mathbb{R}^{n}, \mathfrak{G}\right)$ such that $\operatorname{alt}(T)=0$ is called the first prolongation of the Lie algebra of $G$. Let us denote by $\langle\rangle:, \operatorname{gl}(n, \mathbb{R}) \times \operatorname{gl}(n, \mathbb{R}) \longrightarrow \mathbb{R}$ the Cartan-Killing form, given by $\langle a, b\rangle=\operatorname{trace}(a \cdot b)$. The orthogonal subspace of $\mathfrak{G}$ in $\operatorname{gl}(n, \mathbb{R})$ with respect to this form will be denoted by $\mathfrak{G}^{\perp}$. Our main result is:

Theorem 1.1. The following two assertions are equivalent:

1. For every $G$-structure $P$ over a manifold $M$ there exists a unique connection $\nabla$ adapted to the $G$-structure such that, for every endomorphism $S$ given by a section of the bundle Ad.P and every vector field $X \in \mathfrak{X}(M)$, one has

$$
\operatorname{trace}\left(S \circ i_{X} \operatorname{Tor}(\nabla)\right)=0
$$

Moreover this connection only depends on the first contact of the $G$-structure. ${ }^{2}$ 2. If $T \in \operatorname{Hom}\left(\mathbb{R}^{n}, \mathfrak{G}\right)$ satisfies that

$$
i_{v} \operatorname{alt}(T) \in \mathfrak{G}^{\perp}
$$

for any $v \in \mathbb{R}^{n}$, then

$$
T=0 .
$$

Proof. Let $\mathfrak{G} \subset \operatorname{gl}(n, \mathbb{R})$ be given by the equations

$$
\sum_{i, j} r_{\lambda j}^{i} x_{j}^{i}=0, \quad \lambda=1, \ldots, n^{2}-m
$$

where $m=\operatorname{dim} \mathfrak{G}$, and $\left(x_{j}^{i}\right) \in \operatorname{gl}(n, \mathbb{R})$. Let $e_{1}^{\star}, \ldots, e_{n}^{\star}$ be the standard basis of $\mathbb{R}^{n^{*}}$. The homomorphism $T \in \operatorname{Hom}\left(\mathbb{R}^{n}, \mathfrak{G}\right)$ may be written as

$$
T=\sum e_{i}^{\star} \otimes x_{i}
$$

where $x_{i}=\left(x_{i j}^{k}\right)_{j, k} \in \mathfrak{G}$. Let $\alpha_{1}, \ldots, \alpha_{m}$ be a basis of $\mathfrak{G}$, with $\alpha_{i}=\left(\alpha_{i j}^{k}\right)_{j, k}$. It is readily verified that condition 2) in the statement of the theorem is equivalent to

${ }^{2}$ i.e., if $P$ and $P^{\prime}$ are $G$-structures over $M, \nabla$ and $\nabla^{\prime}$ are their attached connections and $P$ and $P^{\prime}$ have a contact of order one as submanifolds of $L F(M)$ in the fiber of $x \in M$, then $j_{x}^{0} \nabla=j_{x}^{0} \nabla^{\prime}$ 
saying that the following homogeneous linear system of $n^{3}$ equations in $n^{3}$ unknowns only admits the trivial solution:

$$
\left\{\begin{aligned}
\sum_{i, j} \alpha_{s i}^{j}\left(x_{k j}^{i}-x_{j k}^{i}\right) & =0 \\
\sum_{i, j} r_{\lambda j}^{i} x_{k j}^{i} & =0
\end{aligned}\right.
$$

where $i, j, k=1, \ldots, n, s=1, \ldots, m$, and $\lambda=1, \ldots, n^{2}-m$.

Let us prove that condition 2) implies condition 1). To do so, let us first suppose that $\nabla$ and $\hat{\nabla}$ are connections satisfying 1 ). We will see that $\nabla=\hat{\nabla}$. Let $\omega$ and $\widehat{\omega}$ be the connection forms of $\nabla$ and $\widehat{\nabla}$, respectively, and let $\Theta$ and $\widehat{\Theta}$ be their torsion forms. Let $\widetilde{X}_{i}=B\left(e_{i}\right)$ be a basis of the $\nabla$-horizontal distribution in $P$, formed by standard horizontal vector fields. It will be enough to prove that the vector fields $\tilde{X}_{i}$ are also $\widehat{\nabla}$-horizontals. Our hypothesis and the first structure equation imply that

$$
\begin{aligned}
0 & =\sum_{i, j}\left(\Theta^{i}-\widehat{\Theta}^{i}\right)\left(\tilde{X}_{j}, \tilde{X}_{k}\right) \alpha_{s i}^{j} \\
& =\frac{1}{2} \sum_{i, j}\left(\widehat{\omega}_{k}^{i}\left(\widetilde{X}_{j}\right)-\widehat{\omega}_{j}^{i}\left(\tilde{X}_{k}\right)\right) \alpha_{s i}^{j} .
\end{aligned}
$$

Since $\widehat{\omega}\left(\widetilde{X}_{k}\right) \in \mathfrak{G}$, we conclude that $\widehat{\omega}\left(\widetilde{X}_{k}\right)=0$, and so $\nabla=\widehat{\nabla}$.

Now let us see that there exists a connection $\nabla$ satisfying 1$)$. Let $\nabla^{\star}$ be any connection over $P$, and let $\tilde{X}_{i}$ be the associated basis of standard horizontal vector fields. Let us construct the connection $\nabla$ by giving its connection form $\omega$. Again using the first structure equation, we have

$$
\left\{\begin{aligned}
\sum_{i, j} \alpha_{s i}^{j}\left(\omega_{k}^{i}\left(\tilde{X}_{j}^{\star}\right)-\omega_{j}^{i}\left(\tilde{X}_{k}^{\star}\right)\right) & =-2 \sum_{i, j} \alpha_{s i}^{j} \mathrm{~d} \theta^{i}\left(\tilde{X}_{j}^{\star}, \tilde{X}_{k}^{\star}\right), \\
\sum_{i, j} r_{\lambda j}^{i} \omega_{j}^{i}\left(\tilde{X}_{k}^{\star}\right) & =0 .
\end{aligned}\right.
$$

Since our hypothesis imply that this system has a unique solution, we can determine the functions $\omega_{j}^{i}\left(\widetilde{X}_{k}^{\star}\right)$, and it is readily verified that they define a connection form $\omega$ over $P$.

Let us prove now that condition 1) implies condition 2). To do so, it will be enough to prove that condition 1) implies that the system 1.1 only admits the trivial solution. So, let $x_{j k}^{i}$ be a solution of this system, and let $\nabla$ be the connection given by condition 1). Let $\widetilde{X}^{i}$ be its associated basis of horizontal standard vector fields. Let $s: U \subset M \longrightarrow P$ be a section of the $G$-structure, and let us define $\left.\widehat{\omega}_{k}^{i}\left(\tilde{X}_{j}\right)\right|_{\sigma(M)}$ as being the constant functions $x_{j k}^{i}$. It is a straightforward computation to prove 
that we have a linear connection $\widehat{\nabla}$ over $U$, extending $\widehat{\omega}_{k}^{i}\left(\tilde{X}_{j}\right)$ to all $\left.P\right|_{U}$. Let $\widehat{\Theta}$ be its torsion form. We have that

$$
\sum_{i, j}\left(\Theta^{i}-\widehat{\Theta}^{i}\right)\left(\tilde{X}_{j}, \widetilde{X}_{k}\right) \alpha_{s i}^{j}=\frac{1}{2} \sum_{i, j}\left(x_{j k}^{i}-x_{k j}^{i}\right) \alpha_{s i}^{j}=0
$$

and then, using the uniqueness of $\nabla$, we conclude that $\left.\widehat{\omega}_{k}^{i}\left(\widetilde{X}_{j}\right)\right|_{\sigma(M)}=x_{j k}^{i}=0$. This ends the proof.

It should be remarked that the proof of this theorem provides a method of constructing $\nabla$, by solving the system 1.2 .

Definition 1.2. The linear connection $\nabla$ given by 1.1 will be called the canonical connection attached to the $G$-structure.

\section{EXISTENCE OF FUNCTORIAL CONNECTIONS}

If condition 2) of the theorem is satisfied for a Lie group $G$, then the first prolongation of its Lie algebra, $\mathfrak{G}^{(1)}$, vanishes, as it is easily seen. With the additional assumption of the invariance of $\mathfrak{G}$ under transposition, i.e., $\mathfrak{G}^{t}=\mathfrak{G}$, condition 2) of the theorem holds.

Theorem 2.1. If $G \subset \mathrm{GL}(n, \mathbb{B})$ verifies that $\mathfrak{G}^{(1)}=0$ and $\mathfrak{G}$ is invariant under transposition, then condition 2) of the previous theorem holds.

Proof. Let $T \in \operatorname{Hom}\left(\mathbb{R}^{n}, \mathfrak{G}\right)$, with $T\left(e_{k}\right)=x_{k}=\left(x_{k j}^{i}\right)_{i, j}$. We must see that if

$$
\sum_{i, j} \alpha_{i}^{j}\left(x_{k j}^{i}-x_{j k}^{i}\right)=0
$$

for every $\alpha=\left(\alpha_{i}^{j}\right) \in \mathfrak{G}$, then each $x_{k}$ vanishes. Since $\mathfrak{G}^{t}=\mathfrak{G}$, we have that

$$
\sum_{i, j} x_{k j}^{i}\left(x_{k j}^{i}-x_{j k}^{i}\right)=0
$$

and so

$$
\begin{aligned}
\sum_{\substack{i, j, k \\
j \neq k}}\left(x_{j k}^{i}\right)^{2} & =\sum_{i} \sum_{j<k} 2 x_{k j}^{i} x_{j k}^{i} \\
& =\sum_{i} \sum_{j<k}\left(-\left(x_{k j}^{i}-x_{j k}^{i}\right)^{2}+\left(x_{k j}^{i}\right)^{2}+\left(x_{j k}^{i}\right)^{2}\right),
\end{aligned}
$$


and then

$$
\sum_{\substack{i, j, k j \\ j<k}}\left(x_{k j}^{i}-x_{j k}^{i}\right)^{2}=0
$$

therefore

$$
T \in \mathfrak{G}^{(1)}=0
$$

Every Lie subgroup of the orthogonal group $O(n)$ verifies the conditions of this theorem. In the particular case of $G=O(n)$ we get the Levi-Civita connection of the metric structure. However the domain of applicability of Theorem 1.1 includes also subgroups not contained in the orthogonal group. For example, one can check that the Theorem can be applied if $G=\left\{\lambda \cdot I_{n}: \lambda \in \mathbb{R}^{\star}\right\}$ where $I_{n}$ is the identity matrix, or if $G$ is the group formed by exponentiating the Lie algebra

$$
\mathfrak{G}=\left\{\left(\begin{array}{ccc}
0 & a & b \\
b & c & 0 \\
a & 0 & -c
\end{array}\right): a, b, c \in \mathbb{R}\right\}
$$

In the next Theorem we see that the condition $\mathfrak{G}^{(1)}=0$ in Theorem 1.1 is also a necessary condition. Let us note that the connection is not supposed to be adapted to the $G$-structure.

Theorem 2.2. If it is possible to attach a connection to each $G$-structure in a functorial manner with the additional assumption that the connection only depends on the first contact of the $G$-structure then $\mathfrak{G}^{(1)}=0$.

Proof. Let $\alpha=\left(\alpha_{k}^{i j}\right) \in \mathfrak{G}^{(1)}$. The Lie algebra of the group $G^{1}$ of 2-jets of invertible maps with source and target $\mathbb{R}^{n}$ which fixed 0 and preserves the contact up to order 1 of the trivial $G$-structure $P_{0}$ of $\mathbb{R}^{n}$ is given by $\mathfrak{G} \oplus \mathfrak{G}^{(1)}$ (see [5]). Let $f_{t}$ be a curve in $G^{1}$ through the identity at 0 and with tangent vector at 0 is $\alpha$. This curve have the following form in the standard coordinates of $\mathbb{R}^{n}$ :

$$
f_{t}^{i}(x)=\sum_{l} a_{i}^{l}(t) x_{l}+\sum_{j k} a_{i}^{j k}(t) x_{j} x_{k}
$$

hence we have

$$
\begin{aligned}
a_{i}^{l}(0) & =\delta_{i}^{l}, \\
a_{i}^{j k}(0)=\dot{a}_{i}^{l}(0) & =0, \\
\dot{a}_{i}^{j k}(0)+\dot{a}_{i}^{k j}(0) & =\alpha_{i}^{j k} .
\end{aligned}
$$


Suppose now that there exists a functorial assignment of a connection to each $G$ structure Let $\nabla^{t}$ be the connection attached to each $\bar{f}_{t}\left(P_{0}\right)$. Let us denote $X^{i}=$ $\partial / \partial x_{i}$. From the definition of $G^{1}$ and from the dependency of the assignment of the connection on the first contact of the $G$-structures we have that:

$$
\nabla_{f_{t \star}\left(X_{0}^{i}\right)}^{t}\left(f_{t} \cdot X^{j}\right)=\nabla_{f_{t \star}\left(X_{0}^{i}\right)}^{0}\left(f_{t} \cdot X^{j}\right)=f_{t \star}\left(\nabla_{X_{0}^{i}}^{0} X^{j}\right) .
$$

Therefore,

$$
f_{t \star}\left(\nabla_{X_{0}^{i}}^{0} X^{j}\right)=\nabla_{f_{t *}\left(X_{0}^{i}\right)}^{0}\left(f_{t} \cdot X^{j}\right)=\sum_{h} \frac{\partial^{2} f_{t}^{h}}{\partial x_{i} \partial x_{j}}(0) X_{0}^{h}+\nabla_{X_{0}^{i}}^{0} X^{j}
$$

Derivation of this last identity with respect $t$ and evaluating in $t=0$ gives:

$$
0=\alpha_{h}^{i j} X_{0}^{h}
$$

thus finishing the proof.

Theorem 2.3. Suppose that $G$ satisfies the hypothesis of the Theorem 1.1. Then

1. Let

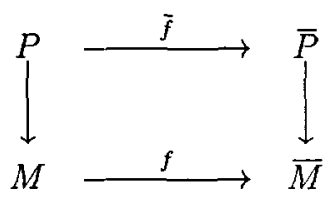

be an isomorphism of G-structures and $\nabla, \bar{\nabla}$ are the canonical connections. Then $f \cdot\left(\nabla_{X} Y\right)=\bar{\nabla}_{f \cdot X} f \cdot Y$ for every $X, Y$ tangent vector fields to $M$.

2. A G-structure is integrable if and only if the tensors of torsion and curvature of the canonical connection vanishes.

Proof. The torsion tensor field of $\nabla$ is $f$-related with the torsion tensor field of $f \cdot \nabla$. From this and from the characterization of the canonical connection readily follows that $\bar{\nabla}=f \cdot \nabla$.

It is a classical result that the it the torsion and the curvature tensors of a connection adapted to a $G$-structure vanish then the $G$-structure is integrable. Conversely, if the $G$-structure is integrable we can choose a section of partials $\left(\partial / \partial x_{1}, \ldots, \partial / \partial x_{n}\right)$ of the bundle of the $G$-structure $P \longrightarrow M$. Let $\nabla^{\star}$ be the connection defined by

$$
\nabla_{\partial / \partial x_{i}}^{\star}\left(\frac{\partial}{\partial x_{j}}\right)=0
$$

This connection is adapted to the $G$-structure and can be used as the auxiliary connection of the proof of Theorem 1.1. But then $\mathrm{d} \theta^{i}\left(\widetilde{X}_{j}^{\star}, \widetilde{X}_{k}^{\star}\right)=0$ and the nonsingular system 1.2 turns to be homogeneous. Hence $\omega_{k}^{i}\left(\tilde{X}_{j}^{\star}\right)=0$ and the canonical connection $\nabla$ coincides with $\nabla^{*}$. 


\section{TWO EXAMPLES}

In the following two subsections are given explicit formulas for the canonical connection attached to almost Hermitian and almost metric contact structures by means of Theorem 1.1. No proof is given. All the formulas can be checked through straightforward although very tidy computations.

3.1. Almost Hermitian structures. Giving an almost Hermitian structure $(J, g)$ on a manifold $M$ is equivalent to reduce the structural group of the tangent bundle to $G=U(n)$. A linear connection $\nabla$ is reducible to a connection adapted to a $U(n)$-structure $P \longrightarrow M$ if and only if $\nabla g=0$ and $\nabla J=0$.

Theorem 1.1 gives a canonical connection attached to each almost Hermitian structure $\nabla$ which can be proved that it is characterized by means of the following two conditions:

1. $\nabla g=0, \nabla J=0$.

2. $g(T(X, Y), Z)-g(T(Z, Y), X)+g(T(J X, Y), J Z)-g(T(J Z, Y), J X)=0$.

It is important to give a formula which enable us to make explicit computations with this connection. This formula is the following:

$$
\begin{aligned}
12 g\left(\nabla_{X} Y, Z\right)= & 6 X g(Y, Z)+2 Y g(Z, X)-2 Z g(X, Y) \\
& -2 J X g(Z, J Y)+2 J Y g(X, J Z)-2 J Z g(X, J Y) \\
& +3 g([X, Y], Z)+2 g([Z, Y], X)-3 g([X, Z], Y) \\
& +2 g([J Z, J Y], X)+g([J X, J Y], Z)-g([J X, J Z], Y) \\
& -g([J X, Y], J Z)+g([J X, Z], J Y)+3 g([J Z, X], J Y) \\
& -3 g([J Y, X], J Z) .
\end{aligned}
$$

The torsion tensor field of the canonical connection of an almost Hermitian structure is also explicitly given by the formula:

$$
\begin{aligned}
12 g(T(X, Y), Z)= & 4 X g(Y, Z)-4 Y g(X, Z)+4 J Z g(Y, J X) \\
& +g(N(Z, Y)+4 J[J Z, Y], X) \\
& +g(N(X, Z)+4 J[X, J Z], Y) \\
& -2 g(N(Y, X)+4[Y, X], Z)
\end{aligned}
$$

where $N$ stands for the Nijenhuis tensor of the almost complex structure $J$.

3.2. Almost contact metric structures. To give an almost contact metric structure $(\varphi, \xi, \eta, g)$ on a manifold $M$ is equivalent to reduce the structural group of the tangent bundle to $G=U(n) \times 1$ [2]. A linear connection $\nabla$ is reducible to a 
connection for a $U(n) \times 1$-structure $P \longrightarrow M$ if and only if $\nabla g=0, \nabla \varphi=0$ and $\nabla \eta=$ 0 . The following 4 properties can be checked by an straightforward computation:

Let $\nabla$ be a connection for the $U(n) \times 1$-structure $P \longrightarrow M$. Then we have, for any vector fields $X, Y$ and $Z$ :

1. The almost contact metric structure is normal if and only if

$$
T(X, Y)-T(\varphi X, \varphi Y)+\varphi T(\varphi X, Y)+\varphi T(X, \varphi Y)=0 .
$$

2. $2 \mathrm{~d} \eta(X, Y)=\eta(T(X, Y))$.

3. If $X$ and $Y$ are vector fields orthogonal to $\xi$, then

$$
\eta\left(\nabla_{X} Y\right)=0 \text {. }
$$

4. $\nabla_{X} \xi=0$.

The canonical connection which is provided by Theorem 1.1 is characterized as follows, as can be easily checked: Given an almost contact metric structure $(\varphi, \xi, \eta, g)$ on $M$, there exists a unique linear connection $\nabla$ satisfying the following two conditions:

1. $\nabla g=0, \nabla \varphi=0$ and $\nabla \eta=0$.

2.

$$
\begin{aligned}
g\left(T\left(\varphi^{2} Z, Y\right), \varphi X\right) & -g\left(T\left(\varphi^{2} X, Y\right), \varphi Z\right)+g(T(\varphi X, Y), \varphi Z) \\
& -g(T(\varphi Z, Y), \varphi X)+2 \eta(X) \mathrm{d} \eta(Z, Y)-2 \eta(Z) \mathrm{d} \eta(X, Y)=0 .
\end{aligned}
$$

The following formulas allow explicit calculations with this canonical connection: With respect to the canonical connection $\nabla$ associated to the almost contact metric structure, we have

1.

$$
\begin{aligned}
4 g\left(\nabla_{\xi} X, Y\right)= & g([Y, \xi]-\varphi[\varphi Y, \xi], X)-g([X, \xi]-\varphi[\varphi X, \xi], Y) \\
& -\eta(X) \eta([Y, \xi])+\eta(Y) \eta([X, \xi]) \\
& -2 \xi(\eta(Y)) \eta(X)+2 \xi(\eta(X)) \eta(Y)+2 \xi g(X, Y)
\end{aligned}
$$

for all $X, Y$ tangent vector fields on $M$.

2 .

$$
\begin{aligned}
12 g\left(\nabla_{X} Y, Z\right)= & 6 X g(Y, Z)+2 Y g(Z, X)-2 Z g(X, Y) \\
& -2 \varphi X g(Z, \varphi Y)+2 \varphi Y g(X, \varphi Z)-2 \varphi Z g(X, \varphi Y) \\
& +3 g([X, Y], Z)+2 g([Z, Y], X)-3 g([X, Z], Y) \\
& +2 g([\varphi Z, \varphi Y], X)+g([\varphi X, \varphi Y], Z)-g([\varphi X, \varphi Z], Y) \\
& -g([\varphi X, Y], \varphi Z)+g([\varphi X, Z], \varphi Y)+3 g([\varphi Z, X], \varphi Y) \\
& -3 g([\varphi Y, X], \varphi Z)
\end{aligned}
$$

for all $X, Y$ and $Z$ tangent vector fields orthogonal to $\xi$. 
Now we can compute the canonical connection $\nabla$ explicitly as follows:

Let $X=\widetilde{X}+\eta(X) \xi, Y=\widetilde{Y}+\eta(Y) \xi, Z=\widetilde{Z}+\eta(Z) \xi$ be tangent vector fields on $M$, where $\tilde{X}, \tilde{Y}, \widetilde{Z}$ are orthogonal to $\xi$. Then using formulas in the beginning of this subsection we get

$$
\begin{aligned}
g\left(\nabla_{X} Y, Z\right)= & g\left(\nabla_{\tilde{X}} Y, Z\right)+\eta(X) g\left(\nabla_{\xi} Y, Z\right) \\
= & g\left(\nabla_{\tilde{X}} \widetilde{Y}, \widetilde{Z}\right)+\eta(X) g\left(\nabla_{\xi} Y, Z\right) \\
& +X(\eta(Y)) \eta(Z)-\xi(\eta(Y)) \eta(X) \eta(Z)
\end{aligned}
$$

and as a consequence of the previous two formulas, the canonical connection $\nabla$ is completely determined.

\section{References}

[1] M.F. Atiyah, R. Bott, V. K. Patodi: On the heat equation and the index theorem. Inventiones Math. 19 (1973), 279-230.

[2] D.E. Blair: Contact manifolds in Riemannian geometry. Lecture Notes in Math., vol 509. Springer, Berlin, 1976.

[3] A. Ferrández, V. Miquel,: Hermitian natural tensors. Math. Scand. 64 (1989), 233-250.

[4] P. B. Gilkey: Local invariants of a pseudo-Riemannian manifold. Math. Scand. 36 (1975), 109-130.

[5] Victor Guillemin: The integrability problem for $G$-structures. Trans. Amer. Math. Soc. 116 (1965), 544-560.

[6] S. Kobayashi and K. Nomizu: Foundations of Differential Geometry I and II. Wiley, New York, 1963 and 1969.

[7] I. Kolár̆, P. Michor and J. Slovák: Natural Operations in Differential Geometry. Springer-Verlag, Berlin, 1993.

[8] A. Valdés: Invariantes diferenciales del fibrado de las referencias proyectivas de una variedad diferenciable y el problema de equivalencia de E. Cartan asociado. Ph. D. Dissertation, Universidad Complutense de Madrid. 1994.

[9] A. Valdés: Differential invariants of $\mathbb{R}^{*}$-structures. Math. Proc. Camb. Phil. Soc. 119 (1996), 341-356.

Authors' addresses: Jose M. Sierra Universidad Alfonso X El Sabio, Apartado de correos 016, 28691 Villanueva de la Cañada, Madrid, Spain; Anton io Valdés, Departamento de Geometría y Topología, Facultad de Ciencias Matemáticas, Univerisad Complutense de Madrid, 28040 Madrid, Spain. 